\title{
Signature of Turbulent Zonal Flows Observed in the DIII-D Tokamak
}

\author{
S. Coda, ${ }^{1, *}$ M. Porkolab, ${ }^{1}$ and K. H. Burrell ${ }^{2}$ \\ ${ }^{1}$ Department of Physics and Plasma Science and Fusion Center, Massachusetts Institute of Technology, \\ Cambridge, Massachusetts 02139 \\ ${ }^{2}$ General Atomics, P.O. Box 85608, San Diego, California 92186-5608
}

(Received 11 September 2000)

\begin{abstract}
The spectrum of turbulent density fluctuations at long poloidal wavelengths in the edge plasma of the DIII-D tokamak peaks at nonzero radial wave number. The associated electric-potential fluctuations cause sheared $\tilde{\mathbf{E}} \times \mathbf{B}$ flows primarily in the poloidal direction. These zonal flows have been predicted by theory and are believed to regulate the overall level of turbulence and anomalous transport. This study provides the first indirect experimental identification of zonal flows.
\end{abstract}

DOI: 10.1103/PhysRevLett.86.4835

Controlled nuclear fusion relies on the efficient confinement of high-temperature plasmas. In tokamaks, the confinement provided by the magnetic-field topology is opposed by outward transport, due to Coulomb collisions and plasma instabilities. While researchers have devised ways to control destructive, large-scale magnetohydrodynamic instabilities, small-scale turbulence is generally present at a level that is sufficient to cause some degree of anomalous transport. However, techniques have also been developed to reduce turbulence and have resulted in the steady improvement of tokamak confinement [1]. Parallel advancements in fluctuation measurement techniques [1] and in the computing power available to numerical simulations [2] have been equally noteworthy and are constantly providing new insight in the properties of turbulence, one of the remaining unsolved problems of classical physics.

The process of turbulence decorrelation and suppression by the sheared $\mathbf{E} \times \mathbf{B}$ flow associated with sheared equilibrium radial electric fields, now well understood theoretically [3], has been identified experimentally as a crucial mechanism in a variety of enhanced-confinement regimes [4]. More recent theoretical and computational work $[2,5-10]$ has led to the important realization that fluctuating sheared poloidal $\tilde{\mathbf{E}} \times \mathbf{B}$ flows, known as zonal flows [11] or radial modes [6], can be driven by microinstabilities and in turn act to regulate them through the same shear stabilization mechanism. In all regimes, therefore, plasma turbulence is expected to be in a self-organized state mediated by zonal flows.

Direct experimental observation of zonal flows is expected to be arduous [12], as it would require extracting the components of the velocity field or electric potential with the required long toroidal and poloidal wavelengths. At present, only indirect signatures can be sought in related physical quantities. In this Letter, we present the first measurement of the fluctuating plasma density in the relevant region of the spectrum. The measurement was performed with a phase-contrast imaging (PCI) diagnostic [13] in the edge region of the Doublet III-D (DIII-D) tokamak. It is shown that the measured component is always characterized by a finite average radial wavelength of the magnitude
PACS numbers: 52.35.Ra, 52.25.Fi, 52.25.Gj, 52.55.Fa

predicted by theory. This implies the existence of electricpotential fluctuations giving rise to turbulent, sheared, primarily poloidal $\tilde{\mathbf{E}} \times \mathbf{B}$ flows. The estimated zonal flow shear is found to be comparable to the dc $\mathbf{E} \times \mathbf{B}$ shear measured in the high-confinement mode ( $H$ mode) [4], and can thus be expected to have a strong regulating effect.

The PCI diagnostic [14] measures the vertical line integrals of the density fluctuations at eight locations distributed along a major radius with a uniform adjustable spacing (typically 0.3 to $0.5 \mathrm{~cm}$ ) and situated in the outer plasma edge of DIII-D $\left(0.80<\psi_{n}<1.17\right.$, where $\psi_{n}$ is the poloidal flux normalized to its value at the last closed flux surface). The measurement accesses frequencies in the range $8-1000 \mathrm{kHz}$ with a sensitivity of $\tilde{n}_{e} \sim 10^{15} \mathrm{~m}^{-3}$.

Owing to the edge location, the line integration spans a small range of magnetic flux, typically corresponding to a radial width of $2-4 \mathrm{~cm}$ on the midplane [15]. Also, the integration occurs nearly tangentially to the magnetic flux surfaces. Thus, wave vectors possessing a significant tangential component will be averaged out [16]. More accurately, the vertical integration restricts the measurement to purely horizontal wave vectors. The components of a horizontal vector in the poloidal magnetic flux coordinates $r$ and $\theta$, respectively normal and tangent to the local flux surface, will satisfy the relation $\left|k_{r}\right| \gg\left|k_{\theta}\right|$, as illustrated in Fig. 1(a) ( $k_{\theta}$ is exactly zero only on the midplane). In addition, the toroidal component $k_{\phi}$ is known to be negligibly small [17]. Thus, the measured spectrum closely approximates the radial spectrum $\left.S\left(k_{r}\right)\right|_{k_{\theta} \sim 0, k_{\phi} \sim 0}$. Extensive analytical and numerical modeling with realistic turbulence spectra $[14,16,18]$ has confirmed this conclusion. A numerical calculation of the responsivity of the system in $\left(k_{r}, k_{\theta}\right)$ space for a typical DIII-D discharge yields the contour plot shown in Fig. 1(b), with the expected anisotropy $\left|k_{r}\right| \gg\left|k_{\theta}\right|$.

To elucidate the spatial structure of the turbulence we examine the spatial correlation function of the PCI signals. This complex Hermitian function, constructed by taking the equal-time correlation coefficient as the real part and its Hilbert transform as the imaginary part, is almost invariably wavelike with a finite spatial period, and decays 

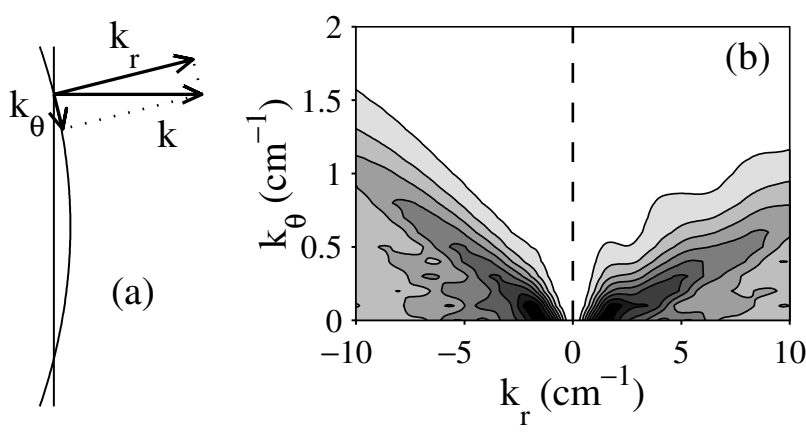

FIG. 1. (a) Decomposition of a horizontal vector into its radial and poloidal components, respectively normal and tangential to the flux surface; each measurement consists of an integration along a vertical line such as that shown in the figure; (b) responsivity of the PCI system as a function of $k_{r}$ and $k_{\theta}$ (darker areas denote larger values); note the different scales for the two axes.

spatially over a finite decay length [14], as shown in Fig. 2. This is true in Ohmic, low-confinement ( $L$ mode), and high-confinement ( $H$ mode) regimes, in a wide range of plasma parameters, and both inside and outside the last closed flux surface (LCFS). The real part is generally larger than the imaginary part, revealing the coexistence of counterpropagating modes, which are statistically uncorrelated, as evidenced by the lack of peaks and nodes in the signal amplitude.

The wave-number spectrum is obtained by Fourier transforming the spatial correlation function and dividing the result by the instrumental transfer function. Spatial antialiasing filtering is effected at the hardware level. Several methods for sparse Fourier transforms have been compared and have yielded similar results, ensuring the robustness of the calculated spectra [14]. The spectrum derived from the data of Fig. 2 is shown in Fig. 3. The spectrum is always double-peaked, with the two peaks at approximately symmetric positive and negative values. Although predominantly inward propagation was initially reported [18], a



FIG. 2. Spatial correlation function of PCI signals: real part (circles), imaginary part (triangles), and envelope (solid curve). The $95 \%$ confidence level is 0.02 . The plot refers to the region between the LCFS and $3 \mathrm{~cm}$ inside, for an Ohmic plasma (toroidal magnetic field $2.1 \mathrm{~T}$, plasma current 1.9 MA, lineaveraged density $3 \times 10^{19} \mathrm{~m}^{-3}$, Ohmic power $3.5 \mathrm{MW}$ ). broader statistical investigation has shown that there can be imbalance in either the inward or outward direction depending on the discharge.

The value of $\left|k_{r}\right|$ at the peaks, $k_{0 r} \neq 0$, which constitutes the key feature of these spectra, can vary between 2 and $7 \mathrm{~cm}^{-1}$ and is well above the instrumental cutoff $(1 / e$ point at $0.65 \mathrm{~cm}^{-1}$ ). The spectra fall sharply to zero for $\left|k_{r}\right|<k_{0 r}$. The frequency spectrum is monotonically decreasing and $k_{0 r}$ increases with frequency.

We have carried out specific simulations to test the interpretation of the observed maxima as nonzero- $k_{r}$ peaks in the $\left.S\left(k_{r}\right)\right|_{k_{\theta} \sim 0}$ spectrum $[14,18]$. In particular, we have attempted to reproduce the measured spectra by postulating a turbulence spectrum peaking at $k_{r}=0$ and $k_{\theta} \neq 0$, as generally found by other measurements $[17,19,20]$. Because of the strongly anisotropic responsivity of PCI [cf. Fig. 1(b)], this is possible only by assuming at once a large ratio $(>3)$ of poloidal to radial correlation lengths, a broad $S\left(k_{\theta}\right)$ spectrum with finite energy at $k_{\theta}=0$, and varying degrees of up-down spatial asymmetry to explain the variation in directionality. However, even under these conditions we could not generate peaks at $k_{r} \neq 0$ at all the radial locations covered by the measurement; also, in up-down asymmetric configurations (single-null diverted plasmas), the calculated locations of the positive and negative peaks are not symmetric. By contrast, the proposed interpretation is consistent with all observations.

Electric-potential fluctuations with $k_{\theta} \sim 0$ and $k_{r} \neq 0$ have been predicted by theory $[10,11,21]$ to be nonlinearly generated by drift waves in an inverse cascade to long poloidal scale lengths. Lacking a radial component, these zonal flows do not cause cross-field transport. Their importance lies in their ability to limit the radial size of the drift-wave eddies through the shear decorrelation mechanism [3] and hence, effectively, to regulate turbulent transport. After zonal flows were properly included in gyrofluid $[5,6,8]$ and gyrokinetic $[2,7,8]$ simulations, it was found that the predicted transport rates decreased by up to a factor of 10. While this work has focused mainly on the ion temperature gradient (ITG) mode as the instability drive,

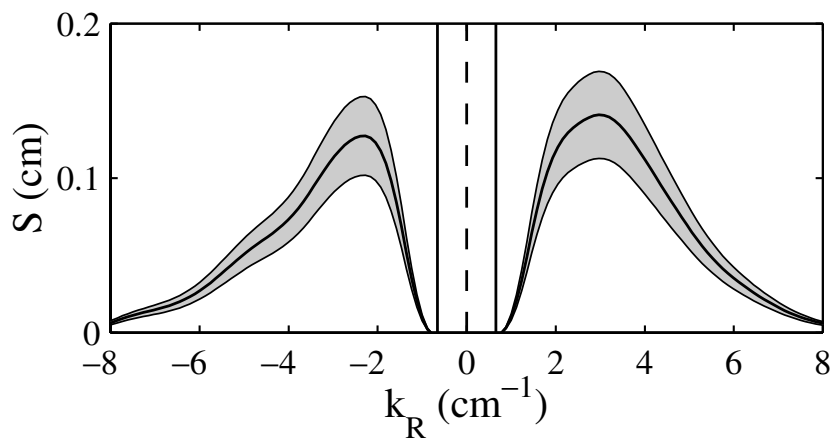

FIG. 3. Radial wave-number spectrum for the case of Fig. 2. The shaded area represents the confidence interval. The spectrum is normalized to an integral of unity and is plotted up to the Nyquist spatial frequency. The blank region near $k=0$ is below the instrumental cutoff. 
which is expected to be dominant in the plasma core, there are also indications $[9,12,22]$ that similar effects should be active in the plasma edge, where resistive ballooning physics may dominate.

These small-scale fluctuating zonal flows are believed to be a general feature of toroidal turbulence [5] and to regulate its saturated state over and above any other turbulence drive or suppression mechanisms that may arise in the plasma. In particular, turbulent zonal flows do not appear to play a specific role in the formation of the edge transport barrier that characterizes the $H$ mode. The $L-H$ transition is a macroscopic state transition, caused by sheared equilibrium dc electric fields. Since the amplitude of the radial modes is a function of the amplitude of the instability drive, the suppression of turbulence that occurs at the transition should cause a corresponding suppression of the nonlinearly generated turbulent flows [6]. Therefore, while the equilibrium sheared flows are larger in $H$ mode, the amplitude of the turbulent zonal flows is expected to be reduced at the $L-H$ transition. This is indeed consistent with our measurements, as we observe a reduction in the turbulence amplitude, as well as a broadening of the $S\left(k_{r}\right)$ spectrum, in going from $L$ to $H$ mode [15].

The response function of the PCI diagnostic [cf. Fig. 1(b)] is such that it should be sensitive to the radial modes but not to the larger finite- $k_{\theta}$ instability drive. By contrast, measurements by other diagnostics integrate over a broad $k_{\theta}$ range and should find a maximum at $k_{r}=0$. This is indeed the case for the majority of tokamak turbulence spectra reported in the literature $[17,19,20]$. There is therefore no contradiction between those measurements and the present ones.

The observed experimental features can be interpreted as a clear signature of zonal flows if the form of the $k_{r}$ spectrum is similar for density and potential fluctuations. This is certainly true if the electron response is adiabatic, as is assumed in the simulations, i.e., if $\tilde{n}_{e} / n_{e}=e[\tilde{\phi}-$ $\left.(\tilde{\phi})_{m=0, n=0}\right] / T_{e}$ [5], where $e$ is the electron charge, $T_{e}$ is the electron temperature, and $m$ and $n$ are the poloidal and toroidal mode numbers, respectively. This also implies that $\tilde{n}_{e}$ is affected only by the sidebands of the dominant $m=0, n=0$ component (azimuthally and poloidally uniform) of the modes [5,6]. Part of our data set lies in a region of low collisionality (the neoclassical plateau regime [23], with normalized electron collisionality $\nu_{* e}$ as low as 1.5), in which the adiabatic approximation is theoretically expected [24] and experimentally known [19] to hold. The observations that the $\left.S\left(k_{r}\right)\right|_{k_{\theta} \sim 0}$ spectrum of $\tilde{n}_{e}$ peaks at finite $\left|k_{r}\right|$ and has vanishing energy for $\left|k_{r}\right| \rightarrow 0$ are absolutely universal and show no dependence on collisionality (in the range $1.5 \leq \nu_{* e} \leq 20$ ). This universality is thus the primary basis for establishing a link between the PCI measurements and the existence of zonal flows. Additionally, simultaneous measurements of the wavenumber spectra of $\tilde{n}_{e}$ and $\tilde{\phi}$ have shown them to be equal in shape [25], even in more collisional regimes, where the parallel dynamics of the electrons become important and an additional nonadiabatic response must be considered. (Indeed, experimental evidence also shows that in this regime $e \tilde{\phi}_{\mathrm{rms}} / T_{e}$ can be up to a factor of 3 higher than $\tilde{n}_{e, \text { rms }} / n_{e}$ [19].)

We are not aware at present of any numerical calculations of the magnitude of the sidebands (to lowest order, the ballooning $m= \pm 1$ components), even though they are qualitatively seen in the simulation results; therefore, we can state only the inequality $e \tilde{\phi}_{\text {rms }} / T_{e}>\tilde{n}_{e, \text { rms }} / n_{e}$, which, as discussed above, would be further enhanced by nonadiabatic effects. Combining PCI estimates of $\tilde{n}_{e}$ with density and temperature measurements by Thomson scattering [14], we can establish an approximate lower bound $\tilde{\phi}_{\text {rms }}>10 \mathrm{~V}$ in Ohmic and $L$ mode plasmas. We then obtain for the zonal-flow shear $d v_{\mathbf{E} \times \mathbf{B}} / d r=c k_{0 r}^{2} \tilde{\phi}_{\text {rms }} / B>$ $2 \times 10^{5} \mathrm{~s}^{-1}$. This lower bound is within an order of magnitude of the value of the macroscopic dc shear measured in $H$ mode [4]. The closeness of the two values is in agreement with gyrokinetic simulations [12]. This is not inconsistent with the dramatic transport suppression that accompanies the $L-H$ transition, since ordered sheared flows are intrinsically more effective than turbulent sheared flows in suppressing turbulence [12].

Predictions for the value of $k_{0 r} \rho_{s}$ (where $\rho_{s}$ is the ion sound gyroradius) vary from 0.1 [12] to 0.6 [6]. In our data set, this parameter varies between 0.2 and 0.4. Also, recently reported gyrokinetic and gyrofluid zonal-flow frequency spectra have $1 / e$ spectral half-widths at $k_{r}=k_{0 r}$ of, respectively, $\Delta f \simeq 4 \times 10^{-4} f_{c i}$ [12], where $f_{c i}$ is the ion cyclotron frequency, and $\Delta f \simeq 10^{-2} v_{t i} / L_{n}$ [26], where $v_{t i}$ is the ion thermal velocity and $L_{n}$ is the density gradient scale length [27]. In a set of $L$ mode plasmas we obtained values of 6 and $7-13 \mathrm{kHz}$, respectively, for these half-widths, to be compared with experimental values in the range $8-15 \mathrm{kHz}$ [14]. In addition, gyrofluid simulations show that the frequency width is an increasing function of $k_{r}$ [26], in agreement with our results. However, such quantitative comparisons may be premature, as numerical work has focused primarily on the plasma core region and on the ITG drive.

Nonlinear effects have been predicted to cause a bursting behavior in the zonal flows over a time scale $1.5 \epsilon \tau_{i i}$ [28], where $\epsilon$ is the inverse aspect ratio $r / R$ and $\tau_{i i}$ is the ion-ion collision time. This time scale is typically in the order of $50-150 \mu \mathrm{s}$. The probability distribution function of the PCI signals is nearly invariably Gaussian for time scales up to $50 \mathrm{~ms}$, whereas an increasingly leptokurtic character (kurtosis $>3$ ) is apparent for intervals exceeding $50 \mathrm{~ms}$, with an asymptotic kurtosis $K \simeq 5.5$ [14]. While the latter can be indicative of intermittent behavior, this occurs over a time scale at least 2 orders of magnitude larger than that predicted by this theory.

In conclusion, the first study of turbulence in the longpoloidal-wavelength region of the spectrum in the outer edge of DIII-D has led to the first observation of the characteristic spectral signature of zonal flows. These flows have been predicted by theory and are believed to be the 
chief regulating mechanism for turbulent transport in tokamaks. The results presented here are consistent with most theoretical predictions. However, further numerical simulations specific to edge conditions are required for a more quantitative comparison.

The authors thank the DIII-D team for the operation of the tokamak, heating systems, and diagnostics. Fruitful discussions with M.A. Beer, T.S. Hahm, and R. E. Waltz are gratefully acknowledged. This work was supported by the U.S. Department of Energy under Grant No. DE-FG02-91ER54109 and Contract No. DE-AC0389ER51114.

*Present and permanent address: Centre de Recherches en Physique des Plasmas, Ecole Polytechnique Fédérale de Lausanne, CH-1015 Lausanne, Switzerland.

Email address: Stefano.Coda@epfl.ch

[1] See B. A. Carreras, IEEE Trans. Plasma Sci. 25, 1281 (1997), and references therein.

[2] Z. Lin et al., Science 281, 1835 (1998).

[3] H. Biglari, P. H. Diamond, and P. W. Terry, Phys. Fluids B 2, 1 (1990).

[4] K. H. Burrell, Phys. Plasmas 4, 1499 (1997).

[5] G. W. Hammett et al., Plasma Phys. Controlled Fusion 35, 973 (1993); M. A. Beer, Ph.D. thesis, Princeton University, 1994.

[6] R.E. Waltz, G. D. Kerbel, and J. Milovich, Phys. Plasmas 1, 2229 (1994).

[7] A. M. Dimits et al., Phys. Rev. Lett. 77, 71 (1996).

[8] G. Rewoldt et al., Phys. Plasmas 5, 1815 (1998).

[9] B. N. Rogers, J. F. Drake, and A. Zeiler, Phys. Rev. Lett. 81, 4396 (1998).

[10] P.H. Diamond et al., in Proceedings of the 17th IAEA Fusion Energy Conference, Yokohama, 1998 (International Atomic Energy Agency, Vienna, Austria, 1999), Vol. 4, p. 1421; L. Chen, Z. Lin, and R. White, Phys. Plasmas 7, 3129 (2000).
[11] A. Hasegawa, C. G. Maclennan, and Y. Kodama, Phys. Fluids 22, 2122 (1979).

[12] T. S. Hahm et al., Plasma Phys. Controlled Fusion 42, A205 (2000).

[13] S. Coda, M. Porkolab, and T.N. Carlstrom, Rev. Sci. Instrum. 63, 4974 (1992).

[14] S. Coda, Ph.D. thesis, Massachusetts Institute of Technology, 1997. Available from http://www.psfc.mit.edu/library/ rr/97rr013_abs.html (pdf) and http://theses.mit.edu:80/ Dienst/UI/2.0/Describe/0018.mit.theses\%2f1997-322 (gif) [accessed March 23, 2001].

[15] S. Coda, M. Porkolab, and K. H. Burrell, Phys. Lett. A 273, 125 (2000).

[16] S. Coda and M. Porkolab, Rev. Sci. Instrum. 66, 454 (1995).

[17] See P.C. Liewer, Nucl. Fusion 25, 543 (1985), and references therein.

[18] S. Coda and M. Porkolab, in Proceedings of the 21th European Conference on Controlled Fusion and Plasma Physics, Montpellier, 1994 (European Physical Society, Petit-Lancy, Switzerland, 1994), Vol. 1, p. 854.

[19] A. J. Wootton et al., Phys. Fluids B 2, 2879 (1990).

[20] R. J. Fonck et al., Phys. Rev. Lett. 70, 3736 (1993).

[21] P. H. Diamond and Y. B. Kim, Phys. Fluids B 3, 1626 (1991).

[22] B. A. Carreras, V. E. Lynch, and L. Garcia, Phys. Fluids B 3, 1438 (1991).

[23] S. P. Hirshman and D. J. Sigmar, Nucl. Fusion 21, 1079 (1981).

[24] M. A. Beer and G. W. Hammett, Phys. Plasmas 3, 4018 (1996).

[25] S. J. Levinson et al., Nucl. Fusion 24, 527 (1984); C. P. Ritz et al., ibid. 27, 1125 (1987); T. Uckan et al., Phys. Fluids B 3, 1000 (1991).

[26] M. A. Beer and G. W. Hammett, in Proceedings of the International Sherwood Fusion Theory Meeting, Los Angeles, 2000 (unpublished).

[27] The different normalization factors $f_{c i}$ and $v_{t i} / L_{n}$ were chosen for the convenience of the respective authors and no fundamental relationship between them is implied.

[28] Z. Lin et al., Phys. Plasmas 7, 1857 (2000). 